\title{
Produção de biomassa de Eucalyptus grandis Hill ex. Maiden em diferentes arranjos de espaçamentos de plantio simples e duplos
}

\author{
Biomass yield of Eucalyptus grandis Hill ex. Maiden under different single and \\ double row planting arrangements
}

\section{Felipe de Córdova Machado', Humberto de Jesus Eufrade-Junior ${ }^{\mathrm{II}}$, Grasiela Spada ${ }^{\mathrm{III}}$,

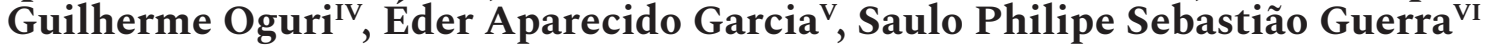

\section{Resumo}

O objetivo deste trabalho foi avaliar o efeito dos espaçamentos de plantio em linhas simples e duplas na área basal, volume de madeira com casca por hectare, densidade básica do tronco e biomassa arbórea de Eucalyptus grandis Hill ex Maiden (clone G-21) manejado no sistema de curta rotação. O ensaio foi composto por quatro espaçamentos de plantio: $3,0 \mathrm{~m} \times 1,0 \mathrm{~m} \times 2,0 \mathrm{~m}$ (linha dupla), $3,0 \mathrm{~m} \times$ $1,0 \mathrm{~m}$ (linha simples), 3,0 $\mathrm{m} \times 1,0 \mathrm{~m} \times 1,0 \mathrm{~m}$ (linha dupla) e $3,0 \mathrm{~m} \times 0,5 \mathrm{~m}$ (linha simples), sendo avaliado aos 12 meses e 24 meses após o plantio. As principais diferenças significativas foram encontradas no segundo período de avaliação para os fatores de variação considerados - espaçamento e idade. A área basal aumentou com o adensamento das florestas, embora o volume não tenha seguido a mesma tendência aos 24 meses de plantio. A densidade básica aumentou com a idade apenas nos espaçamentos simples. No estudo da alocação de biomassa nos diferentes compartimentos da árvore, verificou-se uma maior proporção de biomassa de tronco com a idade, bem como nos espaçamentos mais adensados. Uma das maiores produções florestais $\left(65 \mathrm{Mg} \mathrm{ha}^{-1}\right)$ foi encontrada no espaçamento 3,0 $\mathrm{m} \times 1,0 \mathrm{~m}$ aos 24 meses de idade.

Palavras-chave: Densidade de plantio; Biomassa; Área basal; Florestas energéticas

\begin{abstract}
The objective of this work was to analyze the effect of single and double planting spacing on the basal area, stem wood and bark volume per hectare, stem basic density and above-ground biomass of Eucalyptus grandis Hill ex Maiden (G-21 clone) managed under the short rotation coppice system. Four planting spacings were considered: $3.0 \mathrm{~m} \times 1.0 \mathrm{~m} \times 2.0 \mathrm{~m}$ (double row), $3.0 \mathrm{~m} \times 1.0 \mathrm{~m}$ (single row), $3.0 \mathrm{~m} \times 1.0 \mathrm{~m} \times 1.0 \mathrm{~m}$ (double row) and $3.0 \mathrm{~m} \times 0.5 \mathrm{~m}$ (single row), which were evaluated at 12 months and at 24 months after planting. Main significant differences were found at 12 months after planting for both factors spacing and age. Basal area increased as planting densification, although the volume did not show the same trend at 24 months after planting. Basic density increased as age only at single rows. In the study of the biomass allocation in the different tree compartments, it was verified a higher proportion of stem biomass on older treatments and denser spacings. One of the highest forest productions $\left(65 \mathrm{Mg} \mathrm{ha}^{-1}\right)$ was found in the $3 \mathrm{~m} \times 1 \mathrm{~m}$ planting spacing at 24 months of age.
\end{abstract}

Keywords: Planting density; Biomass; Basal area; Energy forests

Engenheiro Florestal, Me., Duratex Florestal LTDA, Rod. Marechal Rondon, Km 323, zona rural, CEP 17120-000, Agudos (SP), Brasil. felipe_ machado18@hotmail.com (ORCID: 0000-0002-4879-9945)

II Engenheiro Florestal, Dr., Faculdade de Ciências Agronômicas, Universidade Estadual Paulista (UNESP), Av. Universitária, 3780, CEP 18610-034, Botucatu (SP), Brasil. h.eufrade@unesp.br (ORCID: 0000-0002-8402-2260)

III Engenheira Florestal, Drª.., Pesquisadora Autônoma, CEP 17123-054, Agudos (SP), Brasil. gra_spada@hotmail.com (ORCID: 0000-0002-4346-2892) Engenheiro Florestal, Dr., Instituto de Pesquisas e Estudos Florestais, IPEF, Via Comendador Pedro Morganti, 3500, CEP 13415-000, Piracicaba (SP), Brasil. guilherme@ipef.br (ORCID: 0000-0001-9594-8520)

Engenheiro Florestal, Dr., Faculdade de Tecnologia de Ourinhos, FATEC, Av. Vitalina Marcusso, 1400, Campus Universitário, CEP 19910-206, Ourinhos (SP), Brasil, eder.garcia@fatecourinhos.edu.br (ORCID: 0000-0003-4932-5781)

vi Engenheiro Florestal, Dr., Professor da Faculdade de Ciências Agronômicas, Universidade Estadual Paulista (UNESP), Avenida Universitária, 3780, CEP 18610-034, Botucatu (SP), Brasil. saulo.guerra@unesp.br (ORCID: 0000-0002-6760-9094) 


\section{Introdução}

Com a crescente demanda por fontes renováveis de energia, estudos sobre a geração de energia a partir da biomassa florestal têm sido realizados no Brasil e no mundo para geração de energia limpa (MOREIRA, 2011). A madeira pode ser utilizada para abastecimento de fornos, caldeiras em usinas do ramo sucroenergético, indústrias de produção de ração animal, olarias e sistemas de secagem de grãos. No estado de São Paulo, a bioenergia é particularmente estimulada pelo Plano Paulista de Energia, que tem como meta renovar grande parte da matriz energética até o ano de 2020 e as plantações florestais aparecem como uma alternativa (SÃO PAULO, 2012). Também, há uma tendência de aumento de consumo de madeira nos próximos anos pela instalação de usinas termoelétricas e ampliação da indústria de papel, celulose e celulose solúvel no estado.

As espécies de rápido crescimento, como o eucalipto, saem na frente para suprir o fornecimento de madeira em curto período de tempo. Os plantios de eucalipto ocupam 5,67 milhões de hectares de área no país, representando $72,3 \%$ do total de florestas plantadas, e estão localizados principalmente nos estados de Minas Gerais, São Paulo e Mato Grosso do Sul (IBÁ, 2017).

A produtividade média da floresta de eucalipto manejada com ciclo de corte de 6 anos é de $22 \mathrm{t} \mathrm{ha}^{-1} \mathrm{ano}^{-1}$ (BINKLEY et al., 2017) e está entre as maiores do mundo. A floresta pode ser separada em frações ou componentes de biomassa, como os troncos, galhos, folhas, tocos e raízes, ambos podem ser aproveitados para geração de energia (EUFRADE-JUNIOR et al. 2017, SCHEPASCHENKO et al., 2017). Para uso como lenha e carvão, a madeira pode ser utilizada com casca, e em sistemas de curta rotação, o tronco inteiro da árvore é aproveitado (GUERRA et al., 2016).

O espaçamento de plantio tem uma série de implicações do ponto de vista silvicultural, tecnológico e econômico. No manejo florestal, ele pode afetar as taxas de crescimento das plantas, qualidade da madeira, idade de corte, bem como as práticas de exploração e manejo florestal e, consequentemente, os custos de produção (CARON et al., 2015). Para os sistemas de curta rotação de eucalipto são utilizados espaçamentos de $0,5 \mathrm{~m}$ e $1,0 \mathrm{~m}$ entre plantas na linha de plantio e também o uso de linhas duplas, esses espaçamentos são menores daqueles convencionalmente utilizados em plantações comerciais, de 2 a 3 metros entre plantas na linha (GUERRA et al., 2016). O menor espaçamento de plantio acarreta uma diminuição no diâmetro das árvores; entretanto, há uma maior produção de biomassa por hectare (RIBEIRO et al., 2017; ELOY et al., 2018; SCHWERZ et al., 2019).

Quando utilizado para fins de manejo florestal, alguns trabalhos têm demonstrado viabilidade técnica na produção de plantações adensadas, desde que sejam utilizados materiais genéticos selecionados e de maior tolerância à competição (GUERRA et al., 2016; ELOY et al., 2018; SCHWERZ et al., 2019). Contudo, ainda são necessários estudos para avaliar a sustentabilidade dos fatores de crescimento, água, luz e nutrientes no manejo dessas plantações. Por exemplo, Santos et al. (2019) reportaram que a concentração de alocação de nutrientes, bem como biomassa, pode variar nos diferentes genótipos de eucalipto, mesmo em condições edafoclimáticas semelhantes.

Outro parâmetro importante é a densidade da madeira que tem relação direta com produção florestal. Alguns trabalhos têm avaliado a influência do manejo nas características físico-químicas da madeira (GARCIA et al., 2016; MONEDERO et al., 2016; EUFRADE-JUNIOR et al., 2018; ELOY et al., 2018) que são importantes na busca de plantações com alta produtividade e qualidade da biomassa.

Desta forma, o objetivo deste trabalho foi estudar o efeito do arranjo de diferentes espaçamentos de plantio simples e duplos de uma plantação florestal de Eucalyptus grandis Hill ex. Maiden quanto à produção de biomassa arbórea e densidade básica do tronco aos 12 meses e 24 meses de idade. 


\section{Material e métodos}

\section{Localização e caracterização da área de estudo}

O experimento foi instalado na Fazenda Lageado, pertencente a Universidade Estadual Paulista "Júlio de Mesquita Filho" - UNESP, Campus de Botucatu, São Paulo, cujas coordenadas geográficas são $22^{\circ} 50^{\prime} 23^{\prime}$ 'S e $42^{\circ} 25^{\prime} 22^{\prime}$ 'O. O clima predominante no município segundo os critérios adotados por Köppen é Cfa, clima temperado quente (mesotérmico) úmido, com a temperatura média do mês mais quente superior a $22^{\circ} \mathrm{C}$ e o índice pluviométrico médio anual de $1.428 \mathrm{~mm}$ (ALVARES et al., 2014). O tipo de solo da área foi classificado como Nitossolo Vermelho Distroférrico de acordo com o sistema de classificação de solos da EMBRAPA (1999).

\section{Manejo silvicultural}

A implantação do experimento foi realizada em área de aproximadamente um hectare, com o preparo de solo apenas na linha de cultivo, sendo realizada uma subsolagem a $60 \mathrm{~cm}$ de profundidade. Previamente, foi aplicado o herbicida pós-emergente, princípio ativo Glifosato na dose de $2 \mathrm{~kg} \mathrm{ha}^{-1}$.

Para o estudo foi utilizado o clone G-21 de Eucalyptus grandis Hill ex Maiden, o mesmo recebeu adubação de plantio por covetas laterais na muda, utilizando o adubo NPK 7-37-7 com $100 \mathrm{~g} \mathrm{planta}^{-1}$. Também, foram realizadas duas adubações de cobertura, uma aos 90 dias após o plantio e outra aos 360 dias após o plantio. O adubo utilizado foi o NPK 20-0-20 com 150 g planta ${ }^{-1}$.

Após o plantio foi feita a aplicação de herbicida pré-emergente seletivo para eucalipto, princípio ativo isoxaflutole na dose de $150 \mathrm{~g} \mathrm{ha}^{-1}$ na linha de plantio. Foi realizado também o controle de formigas-cortadeiras com o uso de iscas formicidas, princípio ativo sulfluramida, distribuídos de forma sistemática, utilizando $10 \mathrm{~g}$ a cada $5 \mathrm{~m}$ nas entre linhas de plantio.

\section{Delineamento experimental}

Foram instalados diferentes arranjos de plantio variando a relação entre linhas e entre plantas. Ao todo foram utilizados quatro espaçamentos de plantio, conforme pode ser visualizado na Figura 1, sendo que cada parcela tinha uma área de $20 \mathrm{~m} \times 90 \mathrm{~m}\left(1800 \mathrm{~m}^{2}\right)$.

Figura 1 - Espaçamentos de plantio. I - 3,0 $\mathrm{m} \times 1,0 \mathrm{~m} \times 2,0 \mathrm{~m}$ (linha dupla); II - 3,0 $\mathrm{m} \times 1,0 \mathrm{~m}$ (linha simples); III - 3,0 m × 1,0 m × 1,0 m (linha dupla); IV - 3,0 m × 0,5 m (linha simples).

Figure 1 - Planting spacement. I - $3.0 \mathrm{~m} \times 1.0 \mathrm{~m} \times 2.0 \mathrm{~m}$ (double row); II $-3.0 \mathrm{~m} \times 1.0 \mathrm{~m}$ (single row); III $-3.0 \mathrm{~m} \times 1.0 \mathrm{~m} \times 1.0 \mathrm{~m}$ (double row); IV $-3.0 \mathrm{~m} \times 0.5 \mathrm{~m}$ (single row).

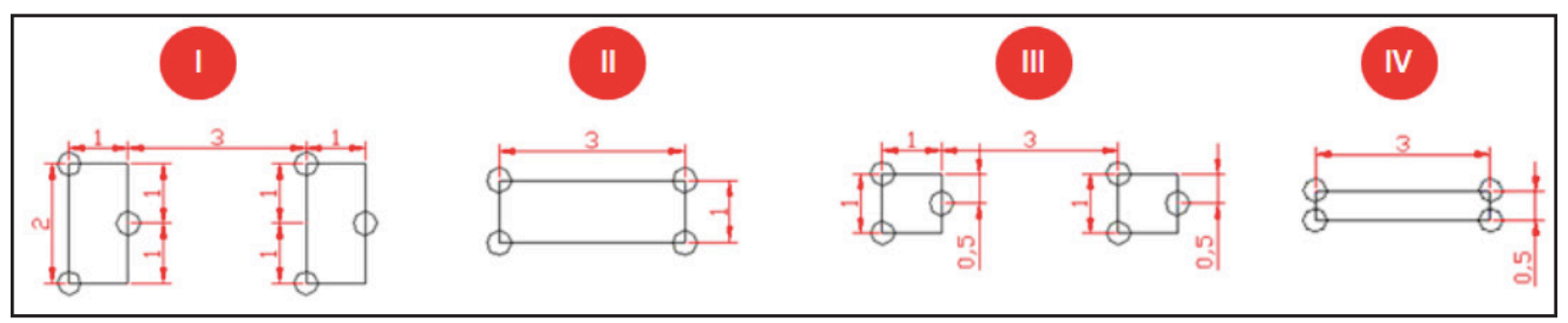

Fonte: Autores (2019)

O experimento foi avaliado aos 12 e 24 meses após o plantio para obtenção da área basal 
por hectare $\left(\mathrm{m}^{2}\right.$ ha $\left.\mathrm{a}^{-1}\right)$, volume de madeira com casca por hectare $\left(\mathrm{m}^{3} \mathrm{ha}^{-1}\right)$, densidade básica do tronco $\left(\mathrm{kg} \mathrm{m}^{-3}\right)$ e biomassa total por hectare $\left(\mathrm{kg} \mathrm{ha}^{-1}\right)$. Foram mensurados os valores de DAP de 30 árvores centrais de cada tratamento com auxílio de uma suta, os resultados encontrados foram multiplicados pela quantidade de árvores para estimativa por hectare (Tabela 1).

Tabela 1 - Área útil, espaçamento de plantio e número de árvores por hectare.

Table 1 - Vital area, planting spacing and density stand.

\begin{tabular}{lccc}
\hline Tratamento & Espaçamento $(\mathbf{m})$ & Área útil $\left(\mathbf{m}^{\mathbf{2}}\right)$ & Árvores ha $^{\mathbf{1}}$ \\
\hline $\mathbf{1}$ & $3 \times 1 \times 2$ & 4,0 & 2500 \\
$\mathbf{2}$ & $3 \times 1$ & 3,0 & 3333 \\
$\mathbf{3}$ & $3 \times 1 \times 1$ & 2,0 & 5000 \\
$\mathbf{4}$ & $3 \times 0,5$ & 1,5 & 6667 \\
\hline
\end{tabular}

Desta forma, foi instalado no campo um delineamento experimental inteiramente casualizado devido à semelhança das condições de sítio do local. Os tratamentos estavam dispostos em esquema fatorial com dois fatores, sendo quatro espaçamentos de plantio e duas idades, totalizando oito tratamentos.

\section{Coleta de árvores-amostra, determinação do volume e da biomassa arbórea}

Para a escolha das árvores-amostra para a cubagem e retirada de discos para a densidade básica do tronco foram consideradas os indivíduos representativos do povoamento, àqueles que apresentavam o diâmetro quadrático médio, conforme a Equação (1):

$$
d_{g}=\sqrt[2]{\frac{\sum d^{2}}{n}}
$$

Em que: $d_{g}$ - diâmetro quadrático médio, em $\mathrm{cm} ; d$ - diâmetro de cada árvore, em $\mathrm{cm} ; n$ - número de árvores.

Quatro árvores-amostra foram escolhidas por tratamento e tiveram seu volume do tronco determinado pela cubagem rigorosa pelo método de Smalian. Os discos utilizados para se determinar a densidade básica da madeira com casca (tronco) foram referentes às posições: $0 \%, 25 \%, 50 \%$ e $75 \%$ da altura total da árvore, os discos tinham cerca de $5 \mathrm{~cm}$ de espessura e os discos coletados na região do topo tinham no mínimo, $3 \mathrm{~cm}$ de diâmetro. A determinação da densidade foi realizada pelo método de imersão em água para cada posição no tronco. A densidade ponderada de cada árvore-amostra foi determinada utilizando a Equação (2).

$$
\rho_{\text {pond }}=\frac{\rho_{1} V_{1}+\rho_{2} V_{2}+\rho_{3} V_{3}+p_{4} V_{4}}{V_{1}+V_{2}+V_{3}+V_{4}}
$$

Em que: $\rho_{b p o n d}$ - densidade básica ponderada do tronco, em $\mathrm{kg} \mathrm{m}^{-3} ; V_{i}$ - volume da seção na posição $i$, em $\mathrm{m}^{3}$; $\rho_{i}$ - densidade básica de cada disco na posição $i$, em $\mathrm{kg} \mathrm{m}^{-3}$. 
A determinação da biomassa foi feita utilizando-se o método direto e destrutivo de árvores. Depois de abatida, cada árvore-amostra teve todas as folhas $\left(\mathrm{P}_{\mathrm{uf}}\right)$ e galhos $\left(\mathrm{P}_{\mathrm{ug}}\right)$ retirados, separadamente toda a biomassa foi pesada no campo para a determinação do peso úmido, com auxílio de uma balança digital móvel com capacidade máxima de $150 \mathrm{~kg}$ e escala de $0,1 \mathrm{~kg}$. Em seguida, foi retirada uma amostra representativa, aproximadamente $200 \mathrm{~g}$ de cada material para determinação da matéria seca.

\section{Análise estatística}

Para cada uma das variáveis mensuradas na biomassa florestal procedeu-se avaliação da homogeneidade da variância e normalidade dos resíduos, a fim de atender ao pré-requisito para análise de variância (ANAVA). Assim, os efeitos das fontes de variação (espaçamentos de plantio e idade) e suas possíveis interações nos tratamentos foram avaliados pela ANAVA, complementada pelo teste de comparação de médias (Tukey), considerados significativos quando $p$-valor $<0,05$.

\section{Resultados e discussão}

\section{Área basal}

Em relação à área basal $(G)$, aos 12 meses, não houve uma tendência de aumento nos espaçamentos com maior densidade inicial de plantio, a maior diferença foi encontrada apenas entre os espaçamentos duplos, sendo que o espaçamento $3,0 \mathrm{~m} \times 1,0 \mathrm{~m} \times 2,0 \mathrm{~m}$ obteve um $\mathrm{G}$ menor do que o espaçamento $3,0 \mathrm{~m} \times 1,0 \mathrm{~m} \times 1,0 \mathrm{~m}$ (Figura 2).

Figura 2 - Área basal Eucalyptus grandis (clone G-21) aos 12 e 24 meses de idade.

Figure 2 - Basal area of Eucalyptus grandis (G-21 clone) at 12 and 24 months of age.

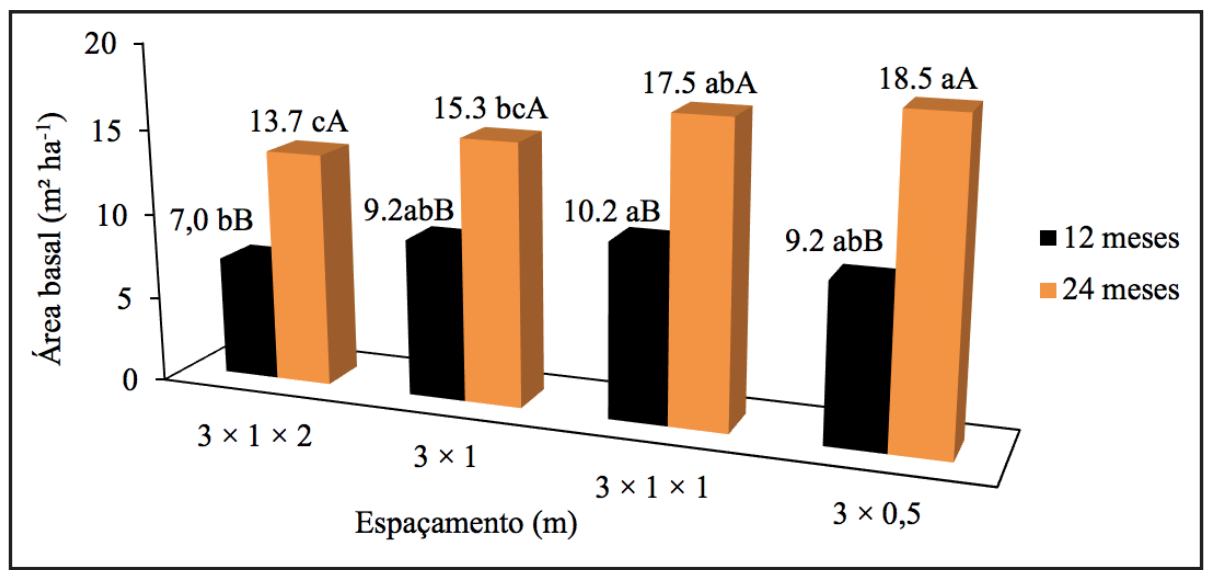

Fonte: Autores (2019)

Em que: Letras maiúsculas iguais entre idades e letras minúsculas iguais entre espaçamentos para a mesma idade, não diferem estatisticamente pelo teste de Tukey $(\alpha=0,05)$.

Where: Capital letters between ages and small letters between spacing for same age do not differ statistically by the Tukey test $(\alpha=0.05)$.

Houve aumento significativo na área basal entre as duas idades analisadas, refletindo o crescimento da espécie e evidenciando que não houve estagnação do crescimento, mesmo no espaçamento mais adensado nos dois primeiros anos após o plantio.

A diferença entre os tratamentos foi maior aos 24 meses de idade, sendo o espaçamento mais 
adensado aquele com maior G. Nesse período, os valores encontrados demonstram a relação direta entre o aumento do número de plantas e a área basal, evidenciando que, apesar dos diâmetros a altura do peito serem maiores nos espaçamentos mais amplos (Tabela 2), eles não foram suficientes para compensar o aumento do número de árvores nas parcelas analisadas. Este padrão também foi encontrado por Ribeiro et al. (2017) estudando florestas clonais de um híbrido interespecífico de Eucalyptus grandis e Eucalyptus urophylla aos 72 meses de idade plantados com área útil variando de $1,50 \mathrm{~m}^{2}$ (6.667 árvores por hectare) até 15,75 $\mathrm{m}^{2}$ (635 árvores por hectare).

Tabela 2 - Diâmetro a altura do peito (DAP) de Eucalyptus grandis (clone G-21).

Table 2 - Diameter at breast height (DBH) of Eucalyptus grandis (G-21 clone).

\begin{tabular}{lcc}
\hline \multirow{2}{*}{ Espaçamento $(\mathbf{m})$} & \multicolumn{2}{c}{ Densidade básica da madeira com casca $\left(\mathbf{k g ~ m}^{-3}\right)$} \\
\cline { 2 - 3 } & $\mathbf{1 2}$ meses & $\mathbf{2 4}$ meses \\
\hline $\mathbf{3} \times \mathbf{1} \times \mathbf{2}$ & $5,8 \mathrm{aB}$ & $8,5 \mathrm{aA}$ \\
$\mathbf{3} \times \mathbf{1}$ & $5,6 \mathrm{aB}$ & $7,8 \mathrm{bA}$ \\
$\mathbf{3} \times \mathbf{1} \times \mathbf{1}$ & $5,1 \mathrm{bB}$ & $6,8 \mathrm{cA}$ \\
$\mathbf{3} \times \mathbf{0 , 5}$ & $4,1 \mathrm{cB}$ & $6,0 \mathrm{dA}$ \\
\hline
\end{tabular}

Em que: Na mesma coluna, médias seguidas de pelo menos uma letra minúscula igual para a análise entre os espaçamentos de plantio, e na mesma linha, médias seguidas de pelo menos uma letra maiúscula igual para a análise entre os períodos de avaliação, não diferem estatisticamente pelo teste Tukey $(\alpha=0,05)$.

\section{Volume}

Na Figura 3 é apresentado o volume de madeira com casca, em $\mathrm{m}^{3} \mathrm{ha}^{-1}$, sendo que, aos 12 meses de idade não foi verificada a influência do espaçamento sobre o volume do tronco, já aos 24 meses somente o espaçamento menos adensado apresentou volume inferior aos demais tratamentos, mesma tendência encontrada por Reiner et al. (2011) estudando Eucalyptus dunnii em diferentes espaçamentos. Quando comparado entre as idades, em todos os espaçamentos ocorreu aumento significativo do volume, evidenciando que não houve restrição de crescimento mesmo nos espaçamentos mais adensados, sugerindo que a competição entre os indivíduos não tenha começado até os dois anos após o plantio, os valores de volume mais que dobraram para todos os espaçamentos.

Em espaçamentos mais adensados existe um aumento da competição por recursos devido ao aumento de número de árvores por hectare, essa competição pode acontecer mais cedo quanto menor for a qualidade do sítio. O crescimento das plantações é alterado também com a idade da floresta, atingindo um pico relativamente cedo seguido por um declínio substancial (TSCHIEDER et al., 2012).

A produção de madeira das árvores depende da absorção de luz, bem como da eficiência na conversão dessa luz em madeira (BINKLEY et al., 2013). As plantações florestais adensadas promovem um maior desenvolvimento do Índice de Área Foliar (IAF) que aumenta a interceptação de luz e fotossíntese, ocasionando uma maior produção de biomassa (SCHWERZ et al., 2019). Com o tempo, as mudanças na estrutura do plantio contribuem para a redução do crescimento, devido ao aumento da diferença na eficiência do uso de recursos entre árvores dominantes e dominadas (CAMPOE et al., 2013), as árvores maiores têm um maior potencial para explorar o solo, além de maior área foliar, maior crescimento de tronco e maior absorção de luz (CHRISTINA et al., 2011; CAMPOE et al., 2013). Entretanto, esse comportamento pode ser minimizado nas plantações adensadas devido ao corte precoce, já que os sistemas de curta rotação de Eucalyptus no Brasil 
são conduzidos em talhadia e a colheita mecanizada pode ser realizada a cada dois anos de idade (GUERRA et al., 2016).

Figura 3 - Produção de madeira com casca por hectare de Eucalyptus grandis (clone G-21) em espaçamentos simples e duplos, aos 12 e 24 meses de idade.

Figure 3 - Stem wood and bark productivity per hectare of Eucalyptus grandis (G-21 clone) at 12 and 24 months of age.

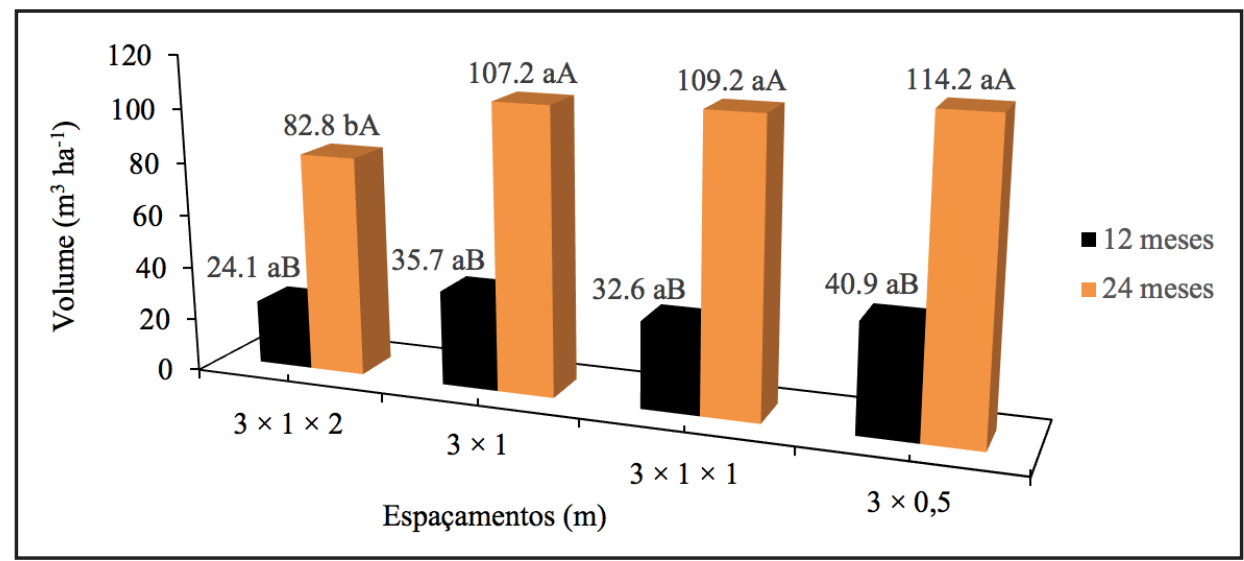

Fonte: Autores (2019)

Em que: Letras maiúsculas iguais entre idades e letras minúsculas iguais entre espaçamentos para a mesma idade, não diferem estatisticamente pelo teste de Tukey $(\alpha=0,05)$.

Where: Capital letters between ages and small letters between spacing for same age do not differ statistically by the Tukey test $(\alpha=0.05)$.

\section{Densidade básica do tronco}

A densidade básica foi influenciada significativamente pelo espaçamento, idade e também a interação entre os dois fatores. Aos 12 meses de idade, o espaçamento $3 \mathrm{~m} \times 0,5 \mathrm{~m}$ apresentou menor valor de densidade básica em comparação aos demais, já aos 24 meses, o espaçamento 3 $\mathrm{m} \times 1 \mathrm{~m}$ apresentou um dos maiores valores de densidade básica (Tabela 3 ).

Em geral, foi encontrada uma maior densidade básica com o aumento do espaçamento de plantio aos 24 meses de idade, o que pode ser explicado pelo crescimento mais lento das árvores, resultando em células com uma parede celular mais espessa. Embora esse comportamento ainda não seja um consenso, considerando-se que existem trabalhos nos quais o espaçamento não interferiu na densidade, como o ensaio realizado por Benin et al. (2017) que encontraram densidades básicas entre 498 e $501 \mathrm{~kg} \mathrm{~m}^{-3}$ para madeiras de Eucalyptus benthamii, em distintos espaçamentos simples, aos 6 anos, na região de Guarapuava - PR. O mesmo aconteceu com Lopes et al. (2017) que não constataram diferença significativa na densidade básica da madeira e da casca em função dos espaçamentos em diferentes clones de Eucalyptus e Corymbia aos 12 meses de idade.

Em relação à idade de avaliação, somente os espaçamentos simples $(3 \mathrm{~m} \times 1 \mathrm{~m}$ e $3 \mathrm{~m} \times 0,5$ m) foram influenciados significativamente, sendo encontrados os maiores valores aos 24 meses nesses espaçamentos. De fato, para a idade, há um consenso que a densidade básica aumenta com a maturidade da árvore como consequência do aumento da espessura da parede celular e diminuição da largura das células (CASTRO et al., 2013). 
Tabela 3 - Densidade básica do tronco de Eucalyptus grandis (clone G-21).

Table 3 - Stem basic density of Eucalyptus grandis (G-21 clone).

\begin{tabular}{lcc}
\hline \multirow{2}{*}{ Espaçamento $(\mathbf{m})$} & \multicolumn{2}{c}{ Densidade básica da madeira com casca $\left(\mathbf{k g ~ m}^{-3}\right)$} \\
\cline { 2 - 3 } & 12 meses & 24 meses \\
\hline $\mathbf{3} \times \mathbf{1} \times \mathbf{2}$ & $352 \mathrm{aA}$ & $361 \mathrm{abA}$ \\
$\mathbf{3} \times \mathbf{1}$ & $351 \mathrm{aB}$ & $379 \mathrm{aA}$ \\
$\mathbf{3} \times \mathbf{1} \times \mathbf{1}$ & $332 \mathrm{aA}$ & $346 \mathrm{bA}$ \\
$\mathbf{3} \times \mathbf{0 , 5}$ & $304 \mathrm{bB}$ & $344 \mathrm{bA}$ \\
\hline
\end{tabular}

Em que: Na mesma coluna, médias seguidas de pelo menos uma letra minúscula igual para a análise entre os espaçamentos de plantio, e na mesma linha, médias seguidas de pelo menos uma letra maiúscula igual para a análise entre os períodos de avaliação, não diferem estatisticamente pelo teste Tukey $(\alpha=0,05)$.

\section{Biomassa total e alocação de biomassa na árvore}

Observa-se que houve aumento da biomassa entre as duas idades analisadas e esta foi significativa em todos os espaçamentos avaliados (Tabela 4). Quando analisado aos 12 meses após o plantio, não foi encontrada diferença significativa da biomassa em função do espaçamento de plantio. Já aos 24 meses, o espaçamento $3 \mathrm{~m} \times 1 \mathrm{~m}$ apresentou um dos maiores valores de biomassa total por hectare.

Tabela 4 - Biomassa total de Eucalyptus grandis (clone G-21).

Table 4 - Biomass yield of Eucalyptus grandis (G-21 clone).

\begin{tabular}{lcc}
\hline \multirow{2}{*}{ Espaçamento $(\mathbf{m})$} & \multicolumn{2}{c}{ Biomassa total $\left(\mathbf{M g ~ h a}^{-1}\right)$} \\
\cline { 2 - 3 } & $\mathbf{1 2}$ meses & $\mathbf{2 4}$ meses \\
\hline $\mathbf{3} \times \mathbf{1} \times \mathbf{2}$ & $17 \mathrm{aB}$ & $50 \mathrm{bA}$ \\
$\mathbf{3} \times \mathbf{1}$ & $21 \mathrm{aB}$ & $65 \mathrm{aA}$ \\
$\mathbf{3} \times \mathbf{1} \times \mathbf{1}$ & $18 \mathrm{aB}$ & $55 \mathrm{abA}$ \\
$\mathbf{3} \times \mathbf{0 , 5}$ & $20 \mathrm{aB}$ & $51 \mathrm{bA}$ \\
\hline
\end{tabular}

Em que: Na mesma coluna, médias seguidas de pelo menos uma letra minúscula igual para a análise entre os espaçamentos de plantio, e na mesma linha, médias seguidas de pelo menos uma letra maiúscula igual para a análise entre os períodos de avaliação, não diferem estatisticamente pelo teste Tukey $(\alpha=0,05)$.

Como regra geral, por um determinado tempo de rotação, um menor espaçamento leva à produção de biomassa total maior por unidade de área (RIBEIRO et a., 2017; ELOY te al., 2018). O espaçamento de plantio simples com menor área útil $(3 \mathrm{~m} \times 0,5 \mathrm{~m})$ apresentou uma das maiores áreas basais $\left(18,5 \mathrm{~m}^{2} \mathrm{ha}^{-1}\right)$ e volume de madeira com casca por hectare $\left(114,2 \mathrm{~m}^{3} \mathrm{ha}^{-1}\right)$ aos 24 meses de idade. Embora, isso não refletiu na maior produção de biomassa por hectare, tal fato pôde ser explicado devido à menor densidade básica do tronco encontrada neste espaçamento de plantio.

Para a alocação de biomassa nos diferentes compartimentos, foi encontrada a seguinte sequência: tronco>galhos>folhas, apenas com exceção no espaçamento $3 \mathrm{~m} \times 1 \mathrm{~m}$, em que foi encontrada uma maior porcentagem de folhas em relação aos galhos (Figura 4). 


\section{Figura 4 - Alocação de biomassa na árvore de Eucalyptus grandis (clone G-21).}

Figure 4 - Tree biomass allocation of Eucalyptus grandis (G-21 clone).

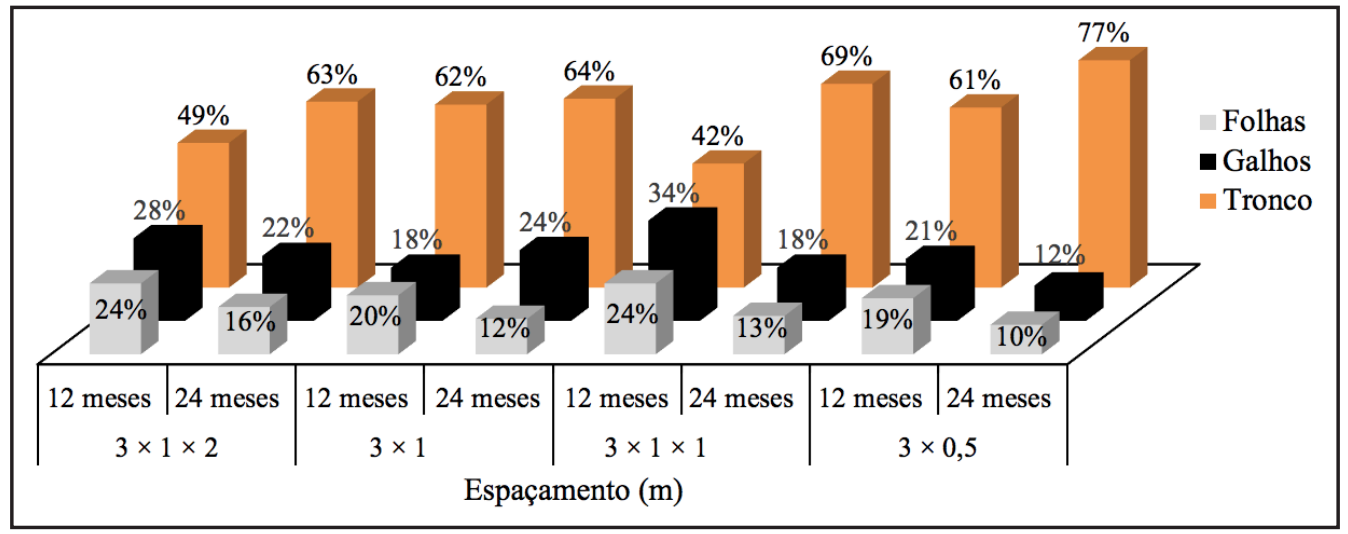

Fonte: Autores (2019)

De forma geral, a alocação de biomassa nas diferentes partes da árvore foi alterada com o espaçamento e a idade. Pode-se notar também que houve em todos os tratamentos um decréscimo da porcentagem de folhas entre 12 e 24 meses, sendo este compensado pelo aumento de biomassa nos galhos e tronco, o mesmo comportamento foi observado por Eloy et al. (2018) avaliando Eucalyptus grandis Hill ex Maiden até os 60 meses de idade.

A proporção de biomassa lenhosa encontrada para os espaçamentos estudados é menor do que o observado para plantações florestais de eucalipto manejados em primeira e segunda rotação com pelo menos seis anos de idade (AZEVEDO et al., 2019). Em árvores mais velhas de eucalipto, a biomassa acima do solo, os troncos, galhos e folhas, possuem uma contribuição de $73,7 \%, 22,3 \%$ e 4,0\%, respectivamente (KUYAH et al., 2013), já que o fechamento do dossel da floresta acentua o crescimento do tronco das árvores na busca de maior luminosidade.

\section{Conclusão}

A área basal $\left(\mathrm{m}^{2} \mathrm{ha}^{-1}\right)$, volume de madeira com casca $\left(\mathrm{m}^{3} \mathrm{ha}^{-1}\right)$, densidade básica do tronco $\left(\mathrm{kg} \mathrm{m}^{-3}\right)$, alocação de biomassa nas árvores e biomassa total $\left(\mathrm{Mg} \mathrm{ha}^{-1}\right)$ foram influenciadas sobretudo pela idade para o clone de Eucalyptus grandis G-21. Os arranjos de espaçamento de plantio mais adensados ocasionaram um aumento da área basal, entretanto, o volume não seguiu a mesma tendência aos 24 meses de plantio.

Nos sistemas estudados não houve uma relação forte do aumento da biomassa com o espaçamento de plantio, já que houve uma diminuição da densidade básica nos tratamentos mais adensados.

A alocação de biomassa apresentou a sequência tronco>galhos $>$ folhas aos 24 meses de avaliação independentemente do espaçamento de plantio. A alocação de biomassa para o tronco aumentou com o adensamento do plantio.

\section{Agradecimentos}

Os autores agradecem ao Laboratório Agroflorestal de Biomassa e Bioenergia (LABB), ao Instituto de Pesquisa em Bioenergia (IPBEN/UNESP), à Coordenação de Aperfeiçoamento de Pessoal de Nível Superior (CAPES) e ao Programa de Pós-graduação em Agronomia - Energia na Agricultura pelo apoio irrestrito para execução do experimento. 


\section{Refêrencias}

ALVARES, C. A. et al. Köppen's climate classification map for Brazil. Meteorologische Zeitschrift, Sttutgart, v. 22, n. 6, p. 711-728, 2013.

AZEVEDO, G. B., et al. Distribuição e produção de biomassa em plantios de eucalipto: um enfoque para a biomassa residual de tocos e raízes. In: GUERRA, S. P. S.; EUFRADE-JUNIOR, H. J. Recuperação energética da biomassa de tocos e raízes de florestas plantadas. Botucatu: FEPAF, 2019. P. 55 - 67.

BENIN, C. C.; WATZLAWICK, L. F.; HILLIG, E. Propriedades físicas e mecânicas da madeira de E. benthamii sob efeito do espaçamento. Ciência Florestal, Santa Maria, RS, v. 27, n. 4, 2017.

BINKLEY D.; CAMPO, O. C.; ALVARES, C.; CARNEIRO, R. L.; CEGATTA, I.; STAPE, J. L.THE interactions of climate, spacing and genetics on clonal Eucalyptus plantations across Brazil and Uruguay. Forest Ecology and Management, Amsterdam, v. 405, p. 271-283, 2017.

BINKLEY D.; CAMPO, O. C.; GSPALTL, M.; FORRESTER, D. I. Light absorption and use efficiency in forests: Why patterns differ for trees and stands. Forest Ecology and Management, Amsterdam, v. 288, P. 5-13, 2013.

CAMPOE, O. C. et al. Stem production, light absorption and light use efficiency between dominant and non-dominant trees of Eucalyptus grandis across a productivity gradient in Brazil, Forest Ecology and Management, Amsterdam, v. 288, n. 1, p. 14-20, 2013.

CARON, B.O. et al. Quantificação da biomassa florestal em plantios de curta rotação com diferentes espaçamentos. Comunicata Scientiae, Bom Jesus, v. 6, p. 106-12, 2015.

CASTRO, A. F. N. M. et al. Análise multivariada para seleção de clones de eucalipto destinados à produção de carvão vegetal. Pesquisa Agropecuária Brasileira, Brasília, v. 48, n. 6, p. 627-635, 2013.

CHRISTINA, M. et al. Almost symmetrical vertical growth rates above and below ground in one of the world's most productive forests. Ecosphere, New York, v. 2, n. 3, p. 1-10, 2011.

ELOY, E.; SILVA, D. A.; CARON, B. O.; ELLI, E. F.; SCHWERZ, F. Effect of age and spacing on biomass production in forest plantations. Revista Árvore, Viçosa, v. 42, p. 01-11, 2018.

EUFRADE-JUNIOR, H.J.; et al. Eucalyptus short-rotation coppice for solid fuel production. Industrial Crops \& Products, Amsterdam, v. 108, p. 636-640, 2017.

EUFRADE-JUNIOR, H.J. et al. Management of Eucalyptus short-rotation coppice and its outcome on fuel quality. Renewable Energy, Oxford, v. 121, p. 309-314, 2018.

EMPRESA BRASILEIRA DE PESQUISA AGROPECUÁRIA - EMBRAPA. Sistema Brasileiro de Classificação de Solos. Rio de Janeiro, Ministério da Agricultura e do Abastecimento, 412p, 1999.

GARCIA, E A.; et al. Análise química da madeira com casca em sistemas florestais de curta rotação. Revista árvore, Viçosa, v. 40, p. 163-171, 2016.

GUERRA, S.P.S. et al. Trade-offs between fuel chip quality and harvesting efficiency in energy plantations, Fuel, London, v. 183, p. 272-277, 2016.

IBÁ. Relatório 2017. Indústria brasileira de árvores, Brasília, 80p., 2017.

KUYAH, S. et al. Allometry and partitioning of above- and Below-ground biomass in farmed eucalyptus species dominant in Western Kenyan agricultural landscapes. Biomass and Bioenergy, United Kingdom, v. 55, n.1, p. 276-284, 2013.

LOPES, E. D.; LAIA, M. L.; SANTOS, A. S.; SOARES, G. M.; LEITE, R. W. P.; MARTINS, N. S. Influencia do espaçamento de plantio na produção energética de clones de Corymbia e Eucalyptus. 
Floresta, Curitiba, v. 47, n. 1, 2017.

MONEDERO, E., et al. Thermochemical and physical evaluation of poplar genotypes as short rotation forestry crops for energy use, Energy Conversion and Management, Amsterdam, v. 129, p. 131-139, 2016.

MOREIRA, J. M. M. A. P. Potencial e participação das florestas na matriz energética. Pesquisa Florestal Brasileira. Colombo, v. 31, n. 68, p. 363-372, 2011.

REINER, D. A.; SILVEIRA, E. R.; SZABO, M. S. O uso do eucalipto em diferentes espaçamentos como alternativa de renda e suprimento da pequena propriedade na região sudoeste do Paraná. Synergismus scyentifica, Pato Branco, v. 6, n. 1, p. 1-7, 2011.

RIBEIRO, M. D. S. B. et al. Avaliação da produção de biomassa do fuste de um clone híbrido de eucalipto sob diferentes espaçamentos. Ciência florestal, Santa Maria, v. 27, p. 31-45, 2017.

SANTOS, K. F.; SCHUMACHER, M. V.; LUDVICHAK A. A.; ARAÚJO, E. F. Biomass and Stock of Nutrients in Different Genotypes of Eucalypts in Southern Brazil. Journal of Experimental Agriculture International, Tarakeswar, v. 34, n. 4, p. 1-12, 2019.

SÃO PAULO. Plano paulista de energia 2020. Secretaria de Energia. São Paulo: PPE2020, 2012.

SCHEPASCHENKO, D. et al. A dataset of forest biomass structure for Eurasia. Scientific Data, London, v. 4, n.170070, 11p., 2017.

SCHWERZ, F.; ELOY, E.; ELLI, E. F.; CARON, B. O. Reduced planting spacing increase radiation use efficiency and biomass for energy in black wattle plantations: Towards sustainable production systems. Biomass and Bioenergy, United Kingdom, v. 120, p. 229-239, 2019.

TSCHIEDER, E. F. et al. Influence of growth dominance and individual tree growth efficiency on Pinus taeda stand growth. A contribution to the debate about why stands productivity declines. Forest Ecology and Management, Amsterdam, v. 277, n. 1, p. 116-123, 2012. 